\title{
Have Imagers Aptly or Inadvertently Overlooked the Neuronal Myocardial Compartment?
}

\author{
Vasken Dilsizian $^{1}$ and Jagat Narula ${ }^{2}$ \\ ${ }^{1}$ University of Maryland School of Medicine, Baltimore, Maryland; and ${ }^{2}$ Icahn School of Medicine at Mount Sinai, New York, New York
}

$\mathbf{I}_{\mathbf{n}}$ equally rewarding. The heart is a constantly moving organ, normally beating 60-100 times a minute and every beat studded with multiphasic physiology. The continuous imaging is pleasing, but quantitative measurements require meticulous gating. Contrastbased imaging requires precise timing to capture contrast transition and a method to preclude contamination from myocardial tracer uptake and to differentiate target uptake from that in the residual blood pool. All the challenges of cardiac imaging have constantly spurred growth in imaging of the cardiovascular system. The contractility of the myocardium is the most prominent trait related to outcomes, and various morphofunctional imaging strategies have focused on muscle mechanics for the assessment of global and regional ejection fraction and of strain and strain rates. Echocardiographic, MR, and radionuclide imaging have performed comparably well. Molecular imaging has targeted muscle ischemia, apoptosis, and necrosis with impressive results. Inducible myocardial ischemia has allowed indirect assessment of significant coronary artery stenosis and has been ably supplemented by CT angiography. The interstitial compartment has predominantly been amenable to MR imaging, which has uniquely addressed the presence of interstitial edema and myocardial fibrosis. The neuronal component of the myocardium, which may directly or indirectly influence the other components or disintegrate because of them, has been sparingly evaluated. This lack of evaluation of the neuronal component may be an inadvertent oversight or an apt omission due to interaction of the neuronal component with abnormalities produced by myocardial compartments that are more easily measured.

The importance of neuronal dysfunction in the progression of heart failure, including adverse outcomes such as life-threatening arrhythmias, is well established $(1,2)$. The long-term pathophysiologic consequences of sympathetic excess include ventricular remodeling, during which the increased norepinephrine concentration in synaptic clefts results in adrenoceptor desensitization, worsening the remodeling process. Improved clinical outcomes associated with adrenergic blocking drugs confirm the centrality of sympathetic dysregulation in heart failure. In the sympathetic nerve terminals, including those in the heart, tyrosine is actively transported and converted to intermediary dopamine, followed by norepinephrine synthesis; norepinephrine is stored in intraneuronal vesicles and

\footnotetext{
Received Apr. 27, 2015; revision accepted Apr. 28, 2015.

For correspondence or reprints contact: Jagat Narula, Icahn School of Medicine at Mount Sinai, One Gustave L. Levy PI., Box 1030, New York, NY 10029.

E-mail: narula@mountsinai.org

COPYRIGHT (c) 2015 by the Society of Nuclear Medicine and Molecular Imaging, Inc.

DOI: $10.2967 /$ jnumed.114.142810
}

released by exocytosis into the synaptic cleft to activate adrenoceptors in the postsynaptic sarcolemmal membrane. In addition, cleft norepinephrine activates presynaptic $\alpha_{2 c}$ receptors to inhibit norepinephrine release. Most of the norepinephrine in the cleft is actively returned back to neurons through human norepinephrine transporter (hNET1), and minimal norepinephrine diffuses into the plasma. The sarcolemma has $\alpha_{(1 a, b, d)^{-}}$and $\beta_{(1-3)^{-}}$-adrenergic receptors; $\beta$ adrenoceptors - through $G_{\alpha s}$ and adenyl cyclase — catalyze adenosine triphosphate to cyclic adenosine monophosphate, and $\alpha$ receptorsthrough $\mathrm{G}_{\alpha q}$-are coupled to phospholipase $\mathrm{C}$ for effector function. Although $\beta$ receptors are associated with positive inotropic, chronotropic, and hypertrophic effects, exposure of $\beta_{1}$ receptors to norepinephrine excess desensitizes them by uncoupling them from transducing $\mathrm{G}$ protein, as mediated by $\beta$-adrenergic receptor kinase 1 and $\beta$ arrestins. The polymorphisms of $\beta_{1}$ receptors $(\operatorname{Arg} 389)$ and $\alpha_{2 c}$ receptors (del 322-325) in heart failure populations have been described as resulting in increased $\beta$-receptor sensitivity and decreased regulatory inhibition of neuronal norepinephrine release. With the supraphysiologic norepinephrine contents of the cleft, in addition to $\beta$-receptor desensitization there is gradual downregulation of hNET1 that adds to norepinephrine toxicity and further obtunds hNET1. When explanted hearts from transplant recipients were compared with unused donor (normal) hearts, hNET1 expression by immunoblot analysis was significantly reduced in failing hearts (Fig. 1). In addition, in paired specimens from patients before and after placement of a left ventricular assist device (LVAD), the post-LVAD samples showed a dramatic increase in hNET1 protein levels (Fig. 2). It is therefore expected that an in vivo imaging strategy for the assessment of hNET1 integrity would mirror remodeling and recovery in heart failure.

This concept of hNET1 downregulation has been best exemplified by radiolabeled metaiodobenzylguanidine (MIBG) imaging (3-6). MIBG is a norepinephrine analog with storage, transport, and reuptake characteristics similar to those of norepinephrine in sympathetic neurons. Downregulation of hNET1 and consequently reduced norepinephrine reuptake is reflected by a low MIBG concentration in the neurons. On the other hand, the increased norepinephrine demand in heart failure and accelerated norepinephrine release lead to an exaggerated rate of MIBG washout. In clinical imaging, radioiodinated MIBG uptake in the failing myocardium has been quantified as the heart-to-mediastinum ratio (with mediastinal uptake presumed to be background uptake). A low ratio (and a high washout rate) in heart failure patients is associated with a worse prognosis, and $\beta$ blockers improve myocardial MIBG retention. Resolution of MIBG uptake correlates with reverse remodeling. A markedly reduced MIBG uptake in end-stage heart failure has also been shown to be reversible by unloading of the LVAD implantation (6). 


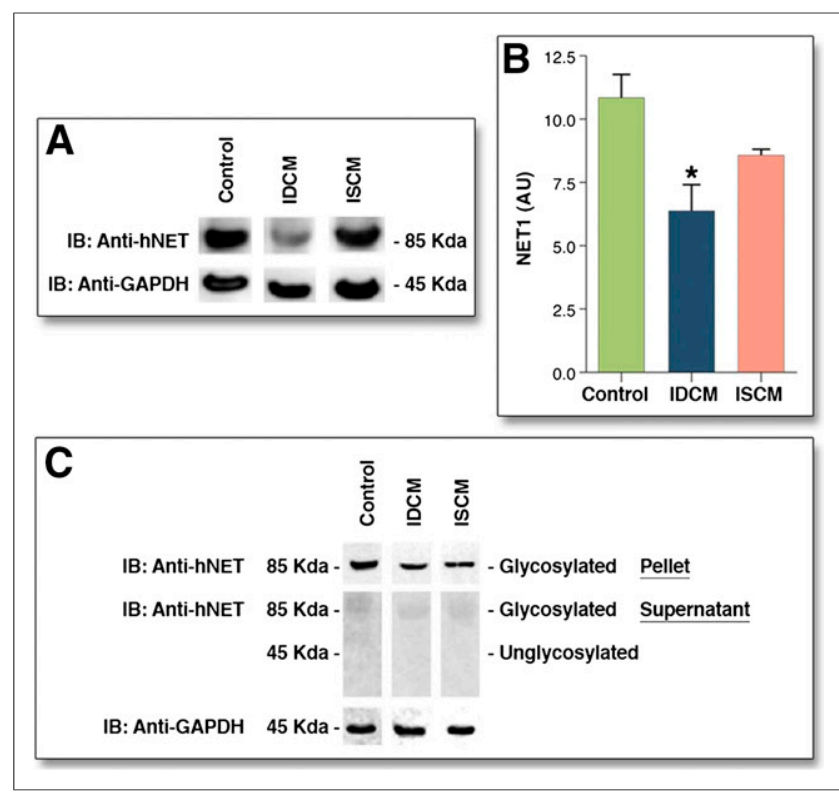

FIGURE 1. hNET1 in failing and control human hearts. (A) Immunoblot analysis of hNET1 expression demonstrates decreased levels in cardiomyopathic hearts. Equal protein in each well was confirmed by glyceraldehyde 3-phosphate dehydrogenase (GAPDH) protein level. (B) Results of densitometric analysis of hNET1 immunoblot (Fluor-S Multilmager; Bio-Rad) are graphed for 4 idiopathic dilated cardiomyopathy hearts (IDCM), 4 ischemic cardiomyopathy hearts (ISCM), and 8 control hearts. Data are mean \pm SEM of intensity of hNET1 bands, in arbitrary units (A.U.), corrected by respective values of GAPDH for each sample. (C) hNET1 glycosylation analysis demonstrates that most hNET1 protein in cardiomyopathic and control human hearts was glycosylated and was bound and precipitated with Con-A Sepharose beads (GE Healthcare) (pellet); barely detectable unglycosylated hNET1 not bound to con-A Sepharose beads remained in supernatant. GAPDH immunoblot confirmed equal protein in each well. ${ }^{*} P<0.05$. (Reprinted with permission of (1).)

In comparison with parasympathetic neurons, sympathetic neurons are evolutionarily primitive (1). Parasympathetic neurons have evolved with cholinesterase protection, but sympathetic neurons rely completely on the neuronal hNET1 reuptake mechanism to manage norepinephrine excess and hence are easily overwhelmed. In the absence of a metabolic inhibitor of norepinephrine in the synaptic cleft, the excess norepinephrine causes adrenoceptor modulation, myocyte apoptosis, and myocellular loss, further worsening myocardial function. The ADMIRE-HF (AdreView Myocardial Imaging for Risk Evaluation in Heart Failure) and ADMIRE-HF extension trials have confirmed the prognostic significance of imaging neuronal dysfunction (2). Although ADMIRE-HF used MIBG imaging, assessment of postsynaptic $\beta$ receptors and presynaptic neuronal norepinephrine synthesis provides additional information about sympathetic integrity.

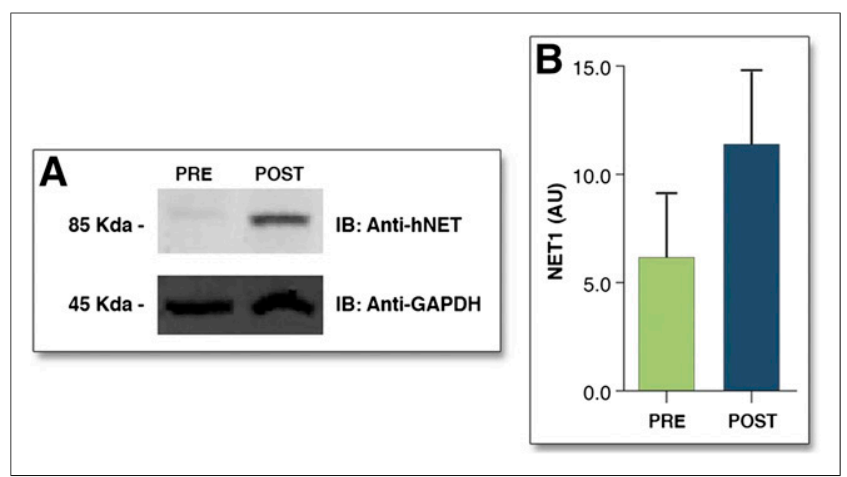

FIGURE 2. hNET1 before and after LVAD placement in human hearts. (A) Immunoblot shows low levels of hNET1 protein in pre-LVAD sample, which are markedly increased in representative post-LVAD sample. Equal protein in each well was confirmed by glyceraldehyde 3-phosphate dehydrogenase (GAPDH) protein level. (B) Densitometric analysis of hNET1 immunoblot (Fluor-S Multilmager; Bio-Rad) for 5 paired pre- and post-LVAD samples shows restoration of hNET1 levels in post-LVAD samples. Data are mean \pm SEM of intensity of hNET1 bands, in arbitrary units (A.U.), corrected by respective values of GAPDH for each sample. (Reprinted with permission of (1).)

The articles in this supplement provide the first comprehensive review of the state of modern imaging of heart failure and neuronal dysfunction. It contains the distilled work of several decades of high-quality laboratory and clinical work.

\section{DISCLOSURE}

No potential conflict of interest relevant to this article was reported.

\section{REFERENCES}

1. Haider N, Baliga RR, Chandrashekhar Y, Narula J. Adrenergic excess, hNET1 down-regulation, and compromised $m$ IBG uptake in heart failure: poverty in the presence of plenty. JACC Cardiovasc Imaging. 2010;3:71-75.

2. Jacobson AF, Senior R, Cerqueira MD, et al. Myocardial iodine-123 metaiodobenzylguanidine imaging and cardiac events in heart failure: results of the prospective ADMIRE-HF (AdreView Myocardial Imaging for Risk Evaluation in Heart Failure) study. J Am Coll Cardiol. 2010;55:2212-2221.

3. Klein T, Abdulghani M, Smith MF, et al. Three-dimensional ${ }^{123}$ I-meta-iodobenzylguanidine cardiac innervation maps to assess substrate and successful ablation sites for ventricular tachycardia: a feasibility study for a novel paradigm of innervation imaging. Circ Arrhythm Electrophysiol. February 23, 2015 [Epub ahead of print].

4. Abdulghani M, Duell J, Smith M, et al. Global and regional myocardial innervation before and after ablation of drug-refractory ventricular tachycardia assessed with ${ }^{123}$ I-MIBG. J Nucl Med. 2015;(6, suppl 4):52S-58S.

5. Dilsizian V, Chandrashekhar Y, Narula J. Introduction of new tests: low are the mountains, high the expectations. JACC Cardiovasc Imaging. 2010;3:117-119.

6. Drakos SG, Athanasoulis T, Malliaras KG, et al. Myocardial sympathetic innervation and long-term left ventricular mechanical unloading. JACC Cardiovasc Imaging. 2010;3:64-70. 